\title{
Modulation of physical understanding by common marmosets (Callithrix jacchus)
}

\author{
Yumiko Yamazaki · Atsushi Iriki · Shigeru Watanabe
}

Received: 23 March 2010 / Revised: 23 August 2010 / Accepted: 27 August 2010 / Published online: 10 September 2010

(C) The Author(s) 2010. This article is published with open access at Springerlink.com

\begin{abstract}
The understanding of physical causality in common marmosets was tested using support problems in which a pair of sheets was presented to determine whether subjects would choose the sheet that had a food item on it (i.e., the sheet was supporting the food item). In two experiments, the conditions were manipulated in terms of the length of the sheet, the distance between the sheet and the food item, the presence of a gap separating the two sheets, and the size of the food item. In Experiment 1, the marmosets had difficulty rejecting an irretrievable food item when it was located closer to them than a retrievable item. Although their performance was strongly affected by the size of the irretrievable food item, they quickly learned to reject that alternative. In contrast, no improvement was found when one sheet was divided into two pieces such that the food item could not be retrieved when its near side was pulled. A similar response tendency was observed in Experiment 2 , in which the effects of the large food item were examined in three different conditions. Thus, common marmosets were influenced by the perceptual features of the food in solving the support problems, as are other
\end{abstract}

Y. Yamazaki $(\bowtie) \cdot S$. Watanabe

The Graduate School of Sociology, Keio University,

2F Mita Toho Building, 3-1-7 Mita, Minato-ku,

Tokyo 108-0073, Japan

e-mail: yumyam@brain.riken.jp

Y. Yamazaki · A. Iriki

Laboratory for Symbolic Cognitive Development,

RIKEN Brain Science Institute 2-1 Hirosawa,

Wako, Saitama 351-0198, Japan

S. Watanabe

Department of Psychology, Faculty of Letters,

Keio University, 2-15-45 Mita, Minato-ku,

Tokyo 108-8345, Japan non-human primates. In addition, they consistently failed to appreciate the presence of a gap and, therefore, failed to reject the distracter alternative. However, all animals rapidly learned that the size of the food item was an irrelevant variable, and some showed an elementary conceptual understanding of support. These findings suggest that marmosets' physical understanding may improve with experience.

Keywords Common marmoset $\cdot$ Contact $\cdot$ Physical causality $\cdot$ Physical support $\cdot$ Problem solving

\section{Introduction}

For animals, understanding the physical causation governing the relationships between objects is a basic and critical cognitive requirement for survival. Such knowledge must be developed rapidly to select a safe route for locomotion, capture prey from a distant position, and find ripe fruits attached to thin branches. Most elementary knowledge about physical causation in humans is evident in early infancy (but see Hood et al. 2000 for contradictory results in infants involving different procedures). At the age of approximately 5 months, human infants can distinguish between possible and impossible relations of support (i.e., whether a box is stable on a platform or insufficiently supported and likely to fall) on the basis of the amount of contact between the objects (e.g., Baillargeon 1994).

The understanding of elementary physical causation has been extensively studied in Piaget's framework. In one task used for examining the understanding of support, an object is placed out of an infant's reach; however, it rests on a cloth that is within the infant's reach (Piaget 1952; Willatts 1984, 1999). The test entails observing whether the infant 
pulls the cloth supporting the object to obtain the object, thereby demonstrating an understanding that the object on the cloth will move closer when the supporting cloth is pulled. In this case, the infant is required to understand that the object is resting on the supporting cloth and would not be pulled closer if it were only in contact with the cloth or covered by the cloth. The mere distance from an actor to an object does not completely decide whether the object is available to the actor; thus, it is necessary to be able to distinguish between a direct visual image of the object in question and the physical mechanisms relevant to the situation before making a decision. Six-month-old infants were able to detect the important features of a support problem by comparing possible and impossible events (Baillargeon 1994; Willatts 1984). This requires the ability to predict the movement of the target object on the basis of physical causality and to detect a discrepancy between predicted and observed events.

Support problems, such as those used by Piaget (1952), have been examined in Asian elephants (Irie-Sugimoto et al. 2008), keas (Auersberg et al. 2009), and non-human primates such as chimpanzees (Mathieu et al. 1980; Spinozzi and Poti 1993), gorillas (Redshaw 1978), and cotton-top tamarins (Hauser et al. 1999, 2002). The results of these studies reveal that these animals have at least an elementary ability to detect relevant physical features by which to differentiate between contact and support.

Animals' level of understanding of the physical causality of support remains controversial. The cotton-top tamarin, a New World monkey, has demonstrated a good transfer of relevant physical features in the means-ends task, which corresponds to the support problem, after extensive training with some examples and independent of various modifications to the material used (Hauser et al. 1999). The animals displayed excellent generalisation performance on untrained problems, and the effect size of generalisation was larger when they were first trained with "connected problems" (i.e., problems in which there was a gap in the unreinforced cloth) than when they were first trained with "on problems" (i.e., in which the food items were either on or not on the cloth). In further research, tamarins with tooluse experience overcame the irrelevant aspects of the task, such as the colour and size of the rewards, unlike those without tool-training experience (Hauser et al. 2002). These results suggest the importance of experience with similar tasks or tasks involving object manipulation such as tooluse training.

In contrast, chimpanzees succeeded in the types of problems given to the tamarins in Hauser et al. (1999) without training (Povinelli 2000). However, through critical contrasts used to examine the understanding of the difference between contact and support, it was observed that chimpanzees attend to specific spatial arrangements of problems and fail to attend to relevant features in the support problem. It was concluded that chimpanzees in this study did not have a concept of support as a form of physical connection, but are able to make fine-scaled perceptual judgements about contact (Povinelli 2000, p. 269, 1. 18-22). If the positive effects of experience with relevant tasks and tool manipulation on support problems also exist for chimpanzees, it is possible that they could overcome the strict conditions in Povinelli (2000) after training because chimpanzees use various tools both in the wild and in captivity (e.g., Matsuzawa 1994).

In the present study, we examined the performance of common marmosets (Callithrix jacchus) on support problems to address questions about their understanding of such problems by systematically manipulating variables that might affect their performance. Common marmosets are small (adults weigh 250-400 g; Araújo et al. 2000) diurnal New World monkeys that originate from Brazilian forests and live in small groups in trees (Abbott and Hearn 1978). They have not been reported to be tool users, although they can be trained to do so (Yamazaki, Watanabe, and Iriki, unpublished data).

To test the understanding of physical causation in common marmosets, we employed problems that compare objects with and without support. The problems were modified from those used in a study involving cotton-top tamarins by Hauser et al. (1999). There are at least three predictions about the performance of the common marmosets. The first model (the conceptual understanding model) hypothesises that they have a sophisticated concept of support and apply it to various conditions, resulting in always choosing an object that supports a food item by rejecting alternatives that have mere contact with food or features that are irrelevant to support. The second model (the perceptual understanding model) argues that they base their responses on perceptual characteristics of the problem, resulting in poor performance when the unsupported food item is located closer to them than the supported one, is larger than the supported one, or is placed on a discontinuous sheet (i.e., beyond the gap). The third model predicts that their performance lies between the predictions of conceptual and perceptual understanding models. In this case, marmosets might have an elementary understanding of support or a fine-grained perceptual strategy based on preferring cloths that perceptually enclose food, similar to the behaviour observed in the chimpanzees (Povinelli 2000). In this case, they would show performance based on the concept of support in some conditions while simultaneously being affected by variables that are essentially irrelevant to the concept of support, such as the sizes of the rewards and the presence of a gap.

A previous report suggested that common marmosets choose the correct alternatives accurately $(89.7 \%$; averaged 
with other conditions that were not support problems) when the correct and incorrect alternatives are equidistant (Spaulding and Hauser 2005). However, whether they succeed in conditions involving the distractor item being located closer to them than the correct item has not been determined. Using experimentally naive common marmosets, the present experiments examined innate physical understanding and changes in performance through defined testing trials to determine the animals' sensitivity to the physical features in question.

\section{Experiment 1}

In Experiment 1, we manipulated the following variables involved in the support problems: (1) length of the supporting sheets, (2) distance between the sheets and the irretrievable food item, (3) gap between the sheets on which the irretrievable food was placed, and (4) size of the irretrievable food item. Using these manipulations, we explored the effects of the distance of the food from the animals, the saliency of the physical and spatial relationships between the materials, the physical continuity of the supporting sheet, and the ability to inhibit selecting the large food item based on the animals' understanding of support.

\section{Methods}

\section{Subjects}

Four adult common marmosets, one male (330 g, M1) and three females (320 g (F1), $450 \mathrm{~g}(\mathrm{~F} 2)$, and $470 \mathrm{~g}(\mathrm{~F} 3))$ were used in the experiments. The animals were all laboratoryborn. The M1 and F1 animals were raised by human caretakers, obtained from CLEA Japan Inc. (Kawasaki, Japan), and tested at Keio University, Tokyo, Japan. They were aged 15 months at the start of testing. F2 and F3 were raised by their parents at the RIKEN Brain Science Institute in Wako, Japan, and were tested there when they were aged 24 and 20 months, respectively. All animals were housed individually in a breeding room on a 12-h (8:00/20:00) light-dark cycle. The temperature and humidity in the breeding room were kept, on average, at $25^{\circ} \mathrm{C}$ and $50 \%$, respectively. The testing sessions were always conducted a few hours before daily feeding. The animals were not deprived of food, and water was freely available in their home cages.

\section{Apparatus}

Identical apparatus and materials were used for the experiments at Keio University and RIKEN. The experimental chamber was $42(\mathrm{~h}) \times 34(\mathrm{w}) \times 35(\mathrm{~d}) \mathrm{cm}$ in size and made of stainless steel. At the bottom of the front panel, there was an aperture $(26(\mathrm{w}) \times 2(\mathrm{~h}) \mathrm{cm})$ through which the subjects could extend their forearms and hands to retrieve the sheets and food items (described below). The aperture was shaped like a comb with $2 \times 5$-cm teeth. The experimental materials, such as the sheets and the food items, were placed on a white acrylic table $(9.5(\mathrm{~h}) \times 35(\mathrm{w}) \times 24(\mathrm{~d}) \mathrm{cm})$, which was connected to the experimental chamber through the aperture. A transparent acrylic board $(0.5(\mathrm{~h}) \times 27(\mathrm{w}) \times$ $14(\mathrm{~d}) \mathrm{cm}$ ) was used to present the available items by sliding it on the table.

Honey cake, which was used as a reinforcer for the marmosets, was cut into tiny portions, shaped into small balls ( $5 \mathrm{~mm}$ in diameter), and served as a standard food item. In the high-incentive condition (large-food (-) and large-food (+) conditions in the procedure), the size of the ball was increased ( $10 \mathrm{~mm}$ in diameter). The sheets were $4 \mathrm{~cm}$ wide and composed of thin black vinyl of varying lengths $(2-10 \mathrm{~cm})$.

Because the subjects were quite nervous when they were alone in the experimental chamber, we placed another marmoset in a cage $(27.5(\mathrm{~h}) \times 23(\mathrm{w}) \times 42(\mathrm{~d}) \mathrm{cm})$ near the experimental chamber. The companion animal could see the experimental marmoset; however, it could not see the details of the experimental conditions because the cage was placed in a lower position than the experimental chamber.

During the experimental sessions, the subjects' behaviour was videotaped with video cameras (HDR-HC9; Sony, Tokyo, Japan) that captured side (from the left) and top views of the subjects and conditions. The videotapes were used for observation of the subjects' behaviour and for scoring after the experiments.

\section{Procedure}

Habituation. To habituate the subjects to the experimental situation and the set-up, we first transferred them from the breeding room to the experimental chamber and fed them with sweets (e.g., honey-cake balls and marshmallows) for several days. Once they consistently came to the experimenter's hand to retrieve the food, the black sheet was introduced. In this phase, the sheets were not presented in pairs, as was the case in the experimental conditions described below; they were presented one at a time with a honey-cake ball resting on it. The subjects were trained to move the sheet to retrieve the food. Thus, there was no need for them to notice a physical relationship between the sheet and the food item. The habituation trials were conducted in up to 3 daily sessions, depending on the skill and nervousness of each subject, but each subject completed less than 40 trials in total.

Support tests. In Experiment 1, there were 30 different configurations of pairs of sheets for which the successful 
response (pulling a sheet with a food item resting on its surface) was reinforced with food, as shown in Fig. 1. They were divided into five categories. Under Standard conditions (problems S1-S8 in Fig. 1), which were identical to the training conditions for the "on problem" in cotton-top tamarins (Hauser et al. 1999), there were pairs of sheets of the same (problems S1-S4) or different (problems S5-S8) lengths. Problems S1 and S2 had two food items, one placed on the sheet and the other off of the sheet. The lengths of the two sheets were identical $(10$ and $5 \mathrm{~cm}$, respectively); therefore, the distracter food item was $1 \mathrm{~cm}$ further away from the subjects than was the retrievable item. The two sheets that appeared in problems S3 and S4 were also identical, but involved no distracter food. The distance between the subject in a choice position (just in front of the aperture of the chamber) and the food was the same for the two sheets in problems S5 and S6 because the length of the sheet with the distracter food was $1 \mathrm{~cm}$ shorter than that of the sheet with the retrievable food item. In the case of problems S7 and S8, the lengths of the two sheets differed $(10$ vs. $5 \mathrm{~cm})$, and the distracter food was placed just off of the nearer sheet (problem S7) or the further sheet (problem S8).
Standard conditions with longer distance (problems S1/Ld-S8/Ld) were identical to those of the Standard conditions S1-S8 (excluding S3 and S4 because there was only one food item in these conditions) except for the distance between the distracter food and the sheet $(2 \mathrm{~cm}$ longer in Longer-distance conditions compared with Standard conditions). By increasing the distance between the food and the animal, it is predicted that the preference for the nearer food will be attenuated and performance will improve. In conditions S1/Ld, S2/Ld, S7/Ld, and S8/Ld, the food item was placed $2 \mathrm{~cm}$ from the sheets. Thus, the total distance between the animal and the distracter food was also increased by $2 \mathrm{~cm}$ compared with the Standard condition. In contrast, in conditions $\mathrm{S} 5 / \mathrm{Ld}$ and $\mathrm{S} 6 / \mathrm{Ld}$, the distance between the distracter food item and the sheet was increased by $2 \mathrm{~cm}$, and the distance between the animal and the correct food and distracter food remained unchanged by decreasing the lengths of the unreinforced sheets by $2 \mathrm{~cm}$ compared with those in problems S5 and S6 under the Standard condition.

For Gap conditions, the unreinforced sheets were separated by a gap of $2 \mathrm{~cm}(\mathrm{G} 1$ and G2) or $3 \mathrm{~cm}(\mathrm{G} 1 / \mathrm{Ld}$ and $\mathrm{G} 2 / \mathrm{Ld})$. For conditions $\mathrm{G} 1$ and G1/Ld, the gap was closer to the subjects than it was for conditions $\mathrm{G} 2$ and $\mathrm{G} 2 / \mathrm{Ld}$.
Fig. 1 Experimental conditions of the sheets in Experiments 1. Problems in Standard, Standard/ Longer-distance, Gap,

Large-food (+), and Large-food

$(-)$ conditions were

experimental conditions in which the marmosets could obtain food if they chose one of the sheets with food on its surface. Problems in the No-reinforcement condition were used as a control for evaluating the animals' choice preference, unexpected cueing by the experimenter, and the effect of the mere presence of the food items. The numbers on each sheet in white represents the length of it in $\mathrm{cm}$. The numbers in red with bidirectional arrows represents the distance between the pieces of sheets, and the sheet and the food item

\section{Standard condition}
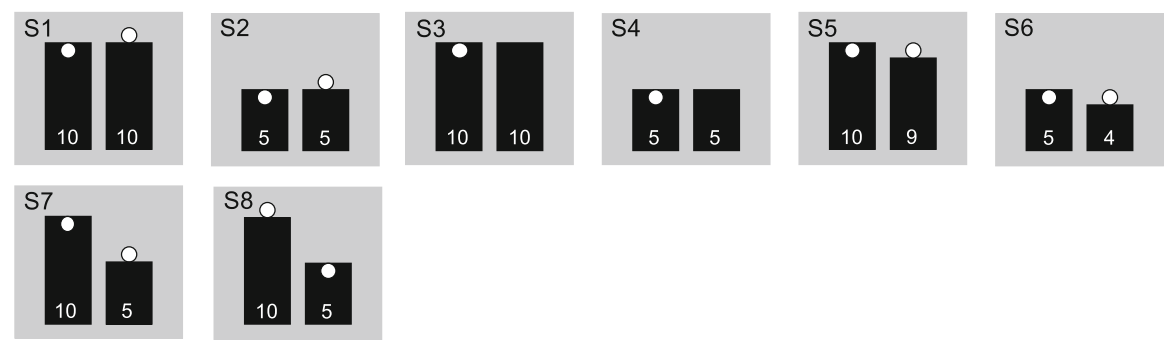

Standard/Longer-distance condition
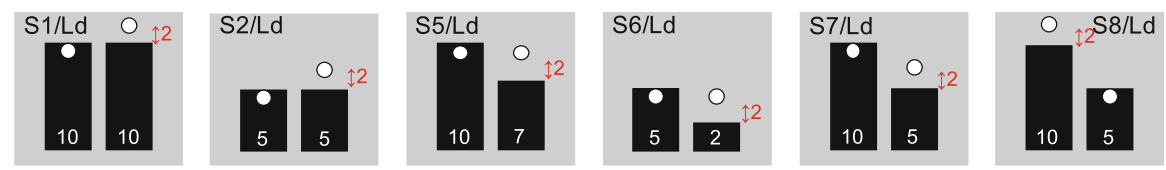

Gap conditions (+Longer distance)

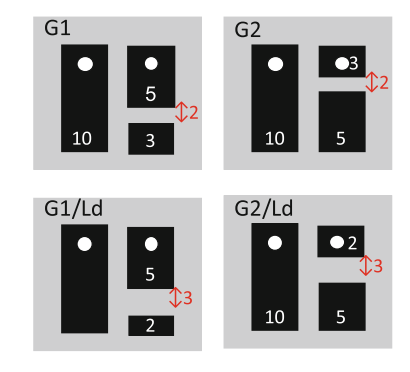

Large-food (-) conditions (+Longer distance)

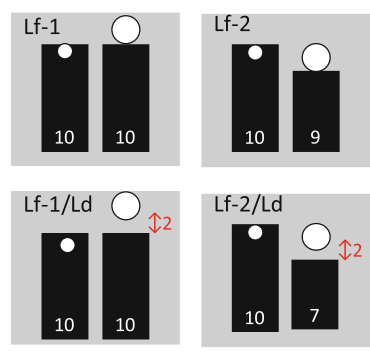

NO-Reinforcement conditions
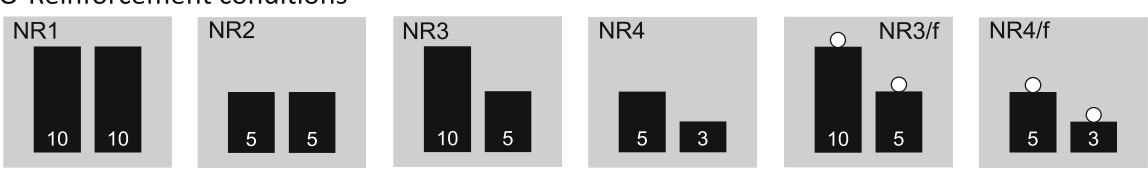
Under Large-food (-) conditions, a larger food item was not retrievable. Problems $\mathrm{Lf}-1$ and $\mathrm{Lf}-2$ were identical to problems S1 and S5 in the Standard condition except that the distracter food was much larger than the retrievable food. For $\mathrm{Lf}-1 / \mathrm{Ld}$ and $\mathrm{Lf}-2 / \mathrm{Ld}$, the distance between the sheet and the irretrievable food was extended from 0 to $2 \mathrm{~cm}$.

As a control, No-reinforcement conditions were introduced to determine whether the animals had a specific or unknown preference for particular experimental materials. In Experiment 1, there were six pairs of sheets that offered no chance to obtain the food. Food was not presented in problems NR1-4, and food was present but not retrievable in problems NR3/f and NR4/f.

Each problem was conducted twice per daily session, and left and right positions of the sheets were counterbalanced. In each session, the problems were randomly interspersed so that the reinforced sheets were not placed in the same right-left position for more than three consecutive trials. The sequence of the problems in each session was also counterbalanced for each subject. Conditions with food reinforcement were conducted in six sessions, resulting in 12 trials per problem in total. Problems in the No-reinforcement condition were conducted in the first three sessions, with six trials per condition in total. The first touch to either of the sheets was defined as a choice response. If an animal touched the correct (reinforced) sheet but did not successfully retrieve the food, it was fed by the experimenter. If it chose the non-reinforced sheet, the trial was ended and the next trial began. When the sheets and the food were presented, they were separated from each other by at least $5 \mathrm{~cm}$. To provide the animals with observation time before making a choice, the stimuli were presented slowly, with
$3 \mathrm{~s}$ allocated to move the materials from the start position to the choice position (where only the sheets were reachable; $5 \mathrm{~cm}$ from the edge of the chamber). During this procedure, the experimenter looked at either of the monitors connected to the video cameras to avoid inadvertent visual cueing of the subjects. If a subject did not choose either of the sheets immediately, the experimenter tapped on the centre of the table to encourage their interest in the materials on the table. If a subject failed to choose within $30 \mathrm{~s}$ after the presentation, the materials were removed. Trials that were skipped were repeated after the planned sequence of problems was completed. The total numbers of repeated trials in Experiment 1 were $1,8,12$, and 0 for $\mathrm{F} 1, \mathrm{~F} 2, \mathrm{~F} 3$, and $\mathrm{M} 1$, respectively. The inter-trial interval was set at a minimum of $10 \mathrm{~s}$, during which the experimenter prepared the materials for the next trial.

\section{Results and discussion}

One experimenter scored all trials, and a second investigator scored one third of the trials from the videotapes to ensure the reliability of scoring. The inter-observer correlation was 1.0 .

The percentage of correct choices is depicted for each subject in Fig. 2. To determine the statistical significance of the animals' choices, the number of correct responses (i.e., responses directed to the sheet with the retrievable food item) in each problem for each animal was compared to the expected value using the binomial test (more than 10 out of 12 trials correct). Subject F2 performed perfectly in the Standard and Longer-distance conditions. The other three subjects performed well, except for problem S7 in the

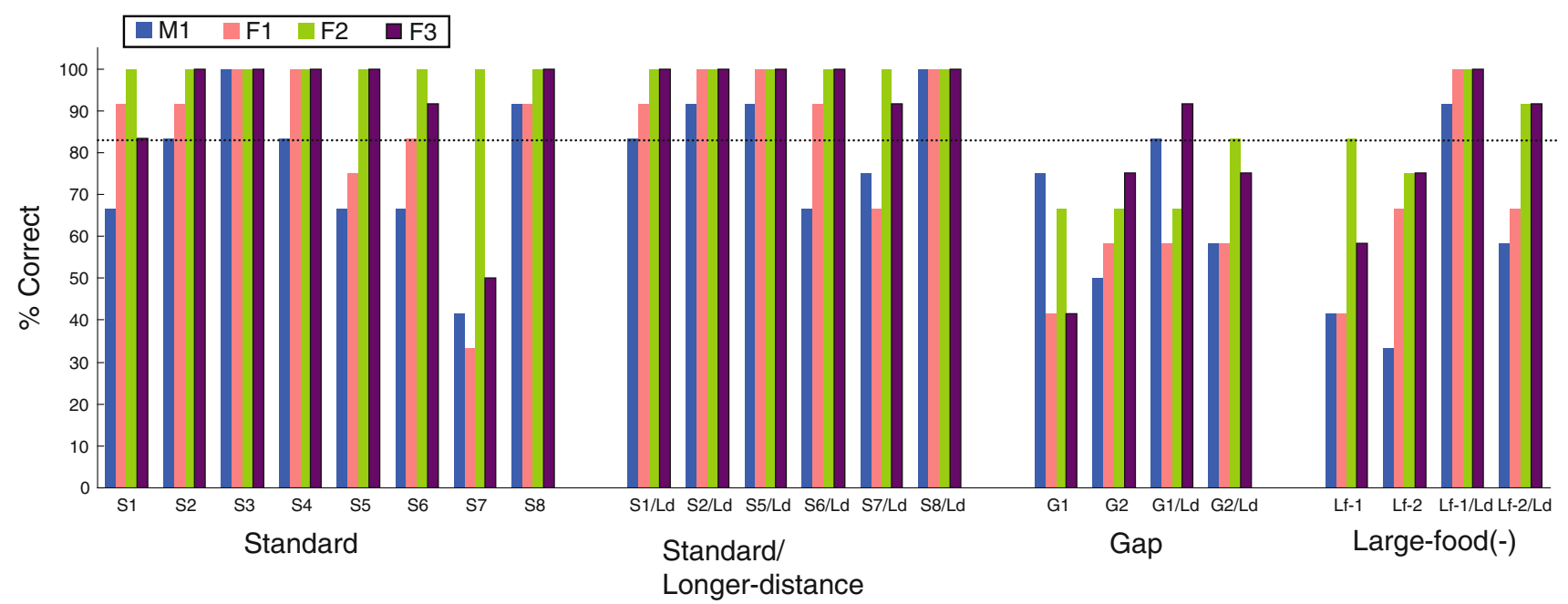

Fig. 2 The percentage of correct choice (i.e., pulling the sheet with a piece of food resting on its surface) in each problem in Standard, Longer-distance, Gap, and Large-food (-) conditions in Experiment 1,

plotted for each subject. The dotted horizontal line indicates the line of significance in the binomial test for 12 test trials 
Table 1 Results of the binomial tests for each problem in each subject in Experiment 1

\begin{tabular}{|c|c|c|c|c|c|c|c|c|c|c|c|c|c|c|}
\hline \multicolumn{9}{|l|}{ Standard } & \multicolumn{6}{|c|}{ Longer-distance } \\
\hline Subject & S1 & S2 & S3 & $\mathrm{S} 4$ & S5 & S6 & S7 & S\& & $\mathrm{S} 1 / \mathrm{Ld}$ & $\mathrm{S} 2 / \mathrm{Ld}$ & $\mathrm{S} 5 / \mathrm{Ld}$ & S6/Ld & $\mathrm{S} 7 / \mathrm{Ld}$ & $\mathrm{S} 8 / \mathrm{Ld}$ \\
\hline M1 & & $*$ & $* *$ & $*$ & & & & $* *$ & $*$ & $* *$ & $* *$ & & & $* *$ \\
\hline F1 & $* *$ & $* *$ & $* *$ & ** & & * & & ** & $* *$ & $* *$ & $* *$ & $* *$ & & $* *$ \\
\hline F2 & $* *$ & $* *$ & ** & $* *$ & $* *$ & ** & $* *$ & ** & $* *$ & $* *$ & $* *$ & $* *$ & $* *$ & ** \\
\hline F3 & $*$ & $* *$ & $* *$ & $* *$ & $* *$ & $* *$ & & $* *$ & $* *$ & $* *$ & $* *$ & $* *$ & $* *$ & $* *$ \\
\hline \multicolumn{9}{|l|}{ Gap } & \multicolumn{6}{|c|}{ Large-food (-) } \\
\hline Subject & \multicolumn{2}{|c|}{ G1 } & \multicolumn{2}{|c|}{ G2 } & G1 & & \multicolumn{2}{|c|}{ G2/Ld } & \multicolumn{2}{|c|}{ Lf -1} & Lf -2 & \multicolumn{2}{|l|}{$\mathrm{Lf}-1 / \mathrm{Ld}$} & $\mathrm{Lf}-2 / \mathrm{Ld}$ \\
\hline M1 & & & & & * & & & & & & & $* *$ & & \\
\hline F1 & & & & & & & & & & & & $* *$ & & \\
\hline $\mathrm{F} 2$ & & & & & & & * & & $*$ & & & $* *$ & & $* *$ \\
\hline F3 & & & & & $* *$ & & & & & & & $* *$ & & $* *$ \\
\hline
\end{tabular}

$* * P<0.01, * P<0.05$

Standard condition (41.7, 33.3, and 50\% for M1, F1, and F3, respectively) (Table 1 and Fig. 2).

When the effect of increasing the distance between the distracter food item and the sheet was determined by comparing the Standard (except for problems S3 and S4, in which there was no distracter) and Longer-distance conditions and pooling all trials in each condition, a paired $t$ test revealed a significant difference between the conditions $\left(t_{3}=5.84, \quad P=0.010\right)$. Thus, increasing the distance between the sheet and the distracter food improved performance. Increasing the distance between the distracter and the sheet location was sufficiently effective for the animals to base their decisions on physical causality, as revealed by the comparison between the Standard and Longer-distance conditions. However, individual differences in improvement in performance between the two conditions were observed. While F3 succeeded on problem S7, F1 could not solve it after lengthening the distance from the non-contacting sheet by $1 \mathrm{~cm}$, and $\mathrm{M} 1$ could not improve his performance on S6/Ld and S7/Ld. Because the increasing saliency of absence of support facilitated performance, perceptual aspects involving physical relationships are an important part of the animals' decision making, in accordance with results obtained in chimpanzees (Povinelli 2000). Even in the case of F2, who performed perfectly in both the Standard and Longer-distance conditions, she might have depended on the perceptual, not conceptual, differences when solving the problems; her performance was at chance in the Large-food (-) condition ( $\mathrm{Lf}-2$ ) but above chance (by a binomial test) when the distance was increased (Lf-2/Ld).

To determine whether there was a learning effect across the sessions (see Fig. 3), one-way ANOVA was conducted

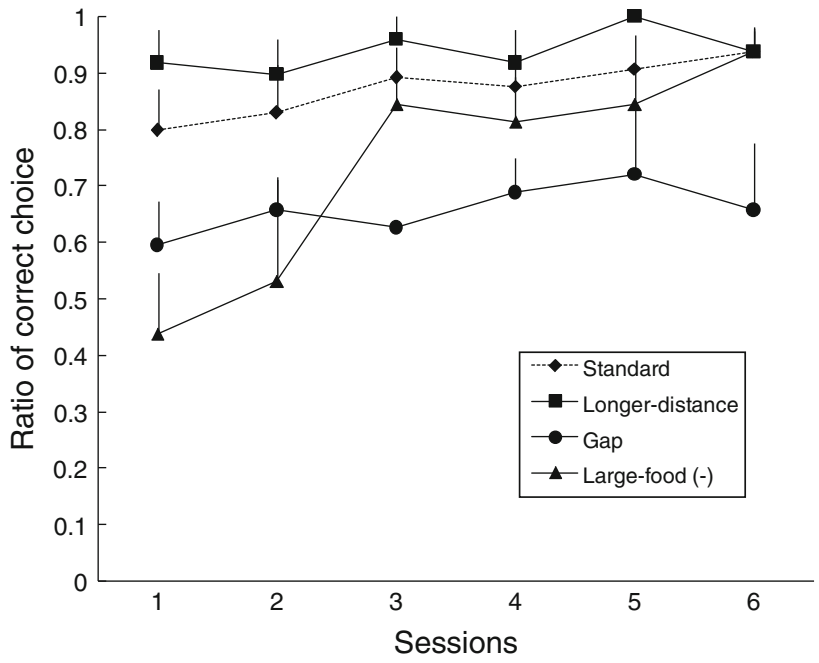

Fig. 3 Averaged percentage of correct choice (i.e., pulling the sheet with a piece of food on its surface) in each test session for each problem in Standard, Longer-distance, Gap, and Large-food (-) conditions in Experiment 1, with standard error

for each type of problem across all subjects. A significant overall effect was found only in the Large-food (-) condition $\left(F_{5,18}=2.77, P=0.012\right)$, and Tukey's HSD test was significant only for the difference between sessions 1 and 6 $(P=0.023, d f=18)$. Learning within the 12 testing trials was observed only in the Large-food $(-)$ condition, not in the Gap condition. In the Gap condition, the animals had to attend to at least two physical characteristics: the support of the food and the separation of a sheet. One possible explanation for their weak performance under the Gap condition is that the marmosets attended mainly to the contact point between the supporting sheets and the food item, not to the point where there was no target (i.e., the gap). 
The proportion of responses in the No-reinforcement condition, in which there was no chance to obtain the food reward, is depicted in Fig. 4. F1 and F2 made few responses to the sheets when there was no food on the table (problems NR1-NR4), whereas M1 showed random responses or position preference in these problems. When the food items were present but not retrievable (problems NR3/f and NR4/f), the subjects showed a preference for shorter sheets, indicating that they chose the alternative in which a food item was located closer to them. Binomial tests revealed a significant difference from the expected results for the NR3/f and NR4/f problems for each subject ( $d f=2, P<0.05$ ), except for subject F2, as shown by the asterisks beside the bars in Fig. 4. Showing no response in the No-reinforcement condition is thought to be an indication of response inhibition. While M1 responded to the sheet in every trial, even when there was no food, the female subjects were capable of inhibiting responses. However, such control was diminished when food items with no physical support were present, as three out of four subjects tended to choose shorter sheets.

To determine whether the subjects ignored the physical relations of support when the irretrievable but nearby food item was present in problem $\mathrm{S} 7$, we conducted a $t$ test to compare the average number of responses to shorter sheets between problems S7 and NR3/f in the No-reinforcement condition. There was a significant difference between the problems $\left(t_{3}=5.42, P=0.012\right)$. A significant difference was also found in the comparison between the average number of responses on problems S7/Ld and NR3/f $\left(t_{3}=5.40, P=0.012\right)$.

Thus, the results for the Standard condition revealed that when two sheets were of the same or similar lengths, the performance of the marmosets was controlled by the physical features of support, not by those of contact. However, when the distracter food was relatively close to the marmosets, their performance was strongly affected, especially when the retrievable food items were located further away from them (problem S7). However, they did not completely ignore the presence of the support, as there was a significant difference between their performance on problems S7 and NR3/f. Problem S7 required the subjects to inhibit a response based on their immediate view of the food and to attend to the relevant physical relationship of support between the sheet and the food. Individual data indicated that, except for F2, the marmosets were affected by the distance of the distracter food item. The results suggested

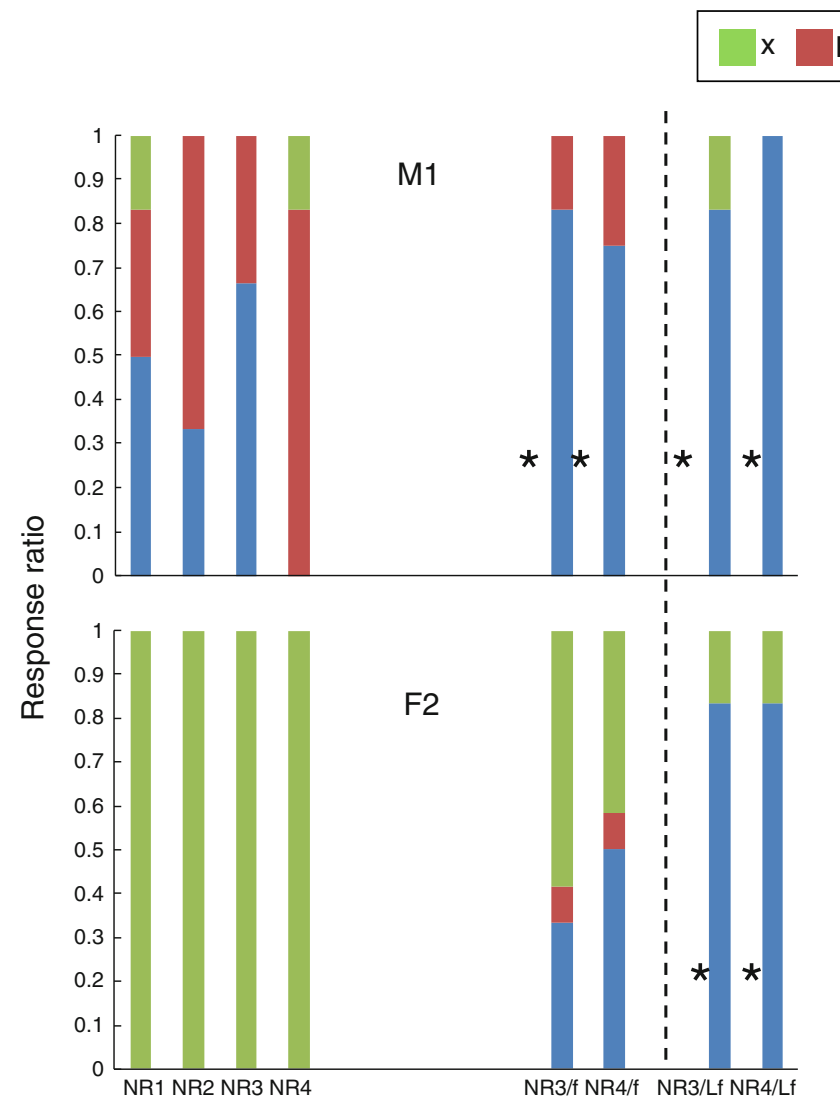

Fig. 4 Proportion of responses for the No-reinforcement condition in Experiments 1 and 2. For problems NR1-NR4, $x$ no response, right choosing right side, and left choosing left side. For problems NR3/f,
Right Left

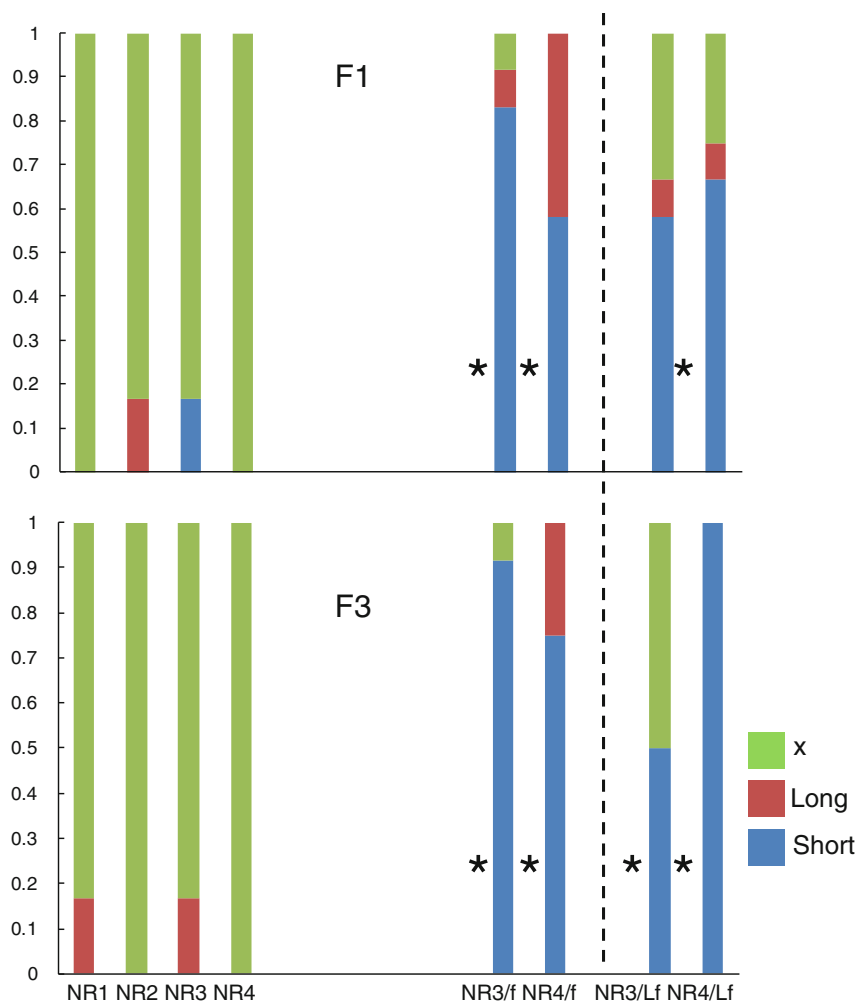

NR4/f, NR3/Lf, and NR4/Lf, $x$ no response, long choosing longer side, and short choosing shorter side. Asterisks indicate that distribution of the responses significantly differed from expected 
that they discriminated between the contact and support conditions. However, unlike the chimpanzees in Povinelli (2000), support was not the primary concept for the marmosets because their use of physical relationships was easily affected by specific contexts, especially the distance between them and the food item.

\section{Experiment 2}

Experiment 2 was conducted to further examine the influences of perceptual cues with a large food item. Additionally, in Experiment 1, the large food item was always presented such that it could not be retrieved ( $\mathrm{Lf}-1, \mathrm{Lf}-2$, $\mathrm{Lf}-1 / \mathrm{Ld}, \mathrm{Lf}-2 / \mathrm{Ld})$. Because the marmosets showed improvement in performance in the Large-food (-) conditions, it is possible that the marmosets considered the large food item as a negative stimulus due to its repetitive appearance as a negative alternative. If so, they should choose an alternative with a small food item in the trials in which the large food item could be retrieved. Thus, in the present experiment, the problems of the Large-food $(+)$ condition, in which larger food items were retrievable, were intermixed to reduce the likelihood of the above outcome.

\section{Methods}

Subjects and apparatus

The same subjects and apparatus as those used in Experiment 1 were used in Experiment 2.

\section{Procedure}

Support tests. Support tests in Experiment 2 included four problems $(\mathrm{Lf}+1, \mathrm{Lf}+2, \mathrm{Lf}+3$, and $\mathrm{Lf}+4)$ for the Large-food $(+)$ condition, four problems for the Large-food $(-)$ condition (Lf-3, Lf-4, Lf-5, Lf-6), and two problems for the Gap/Large-food (-) condition (G1/Lf- and G2/Lf-), as depicted in Fig. 5. In the Large-food (+) condition, the retrievable food was twice as large in diameter (i.e., $10 \mathrm{~mm}$ in diameter) as the typical food size (i.e., $5 \mathrm{~mm}$ in diameter). The $\mathrm{Lf}+3$ and $\mathrm{Lf}+4$ problems were similar to problems Lf- 1 and Lf-2 for the Large-food (-) condition in Experiment 1 except that the sizes of the food items were reversed on reinforced or unreinforced sheets. For the Large-food (-) condition, problems $\mathrm{Lf}-3, \mathrm{Lf}-4, \mathrm{Lf}-5$, and $\mathrm{Lf}-6$ were similar to problems S5, S6, S7, and S8 in the Standard condition, except for the size of the distracter food. Problems G1/Lf - and G2/Lf- in the Gap/Large-food (-) condition were similar to problems G1 and G2 in the Gap condition in Experiment 1, except for the presence of
Large-food(+) conditions

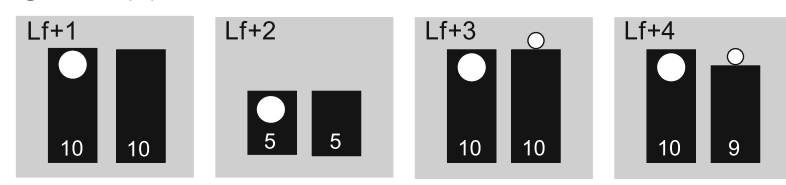

Large-food(-) conditions

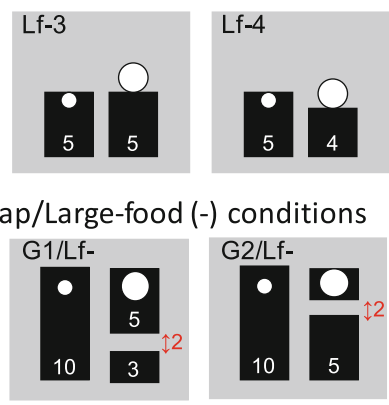

NO-Reinforcement/Large-food conditions

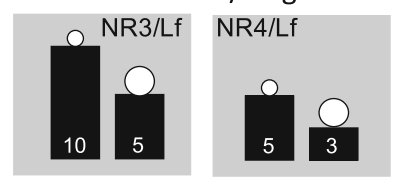

Fig. 5 Experimental conditions of the sheets in Experiments 2. Problems in Large-food (+), Large-food (-), and Gap/Large-food (-) conditions were experimental conditions in which the marmosets could obtain food if they chose one of the sheets with food on its surface. Problems in the No-reinforcement condition were used as a control for evaluating the animals' choice preference, unexpected cueing by the experimenter, and the effect of the mere presence of the food items. The numbers on each sheet in white represents the length of it in $\mathrm{cm}$. The numbers in red with bidirectional arrows represents the distance between the pieces of sheets

the large food items on the far side of the unreinforced sheet. Additionally, two control problems (NR3/Lf and NR4/Lf in Fig. 5) from the No-reinforcement condition were used to examine impulsive choices caused by the presence of large food items. Each problem was tested 12 times in three daily sessions, each of which contained 24 trials. The left and right positions were counterbalanced, resulting in each problem being tested six times with the same configuration. Similar to Experiment 1, if a subject failed to choose within $30 \mathrm{~s}$ after a problem's presentation, the materials were removed. Trials that were skipped were conducted again after the planned sequence of problems was completed. The total numbers of rerun trials in Experiment 2 were $10,16,7$, and 1 for F1, F2, F3, and M1, respectively. The inter-trial interval was set at a minimum of $10 \mathrm{~s}$, during which the experimenter prepared the materials for the next trial.

\section{Results and discussion}

The percentage of correct choices for each subject is depicted in Fig. 6. All subjects performed perfectly on each 


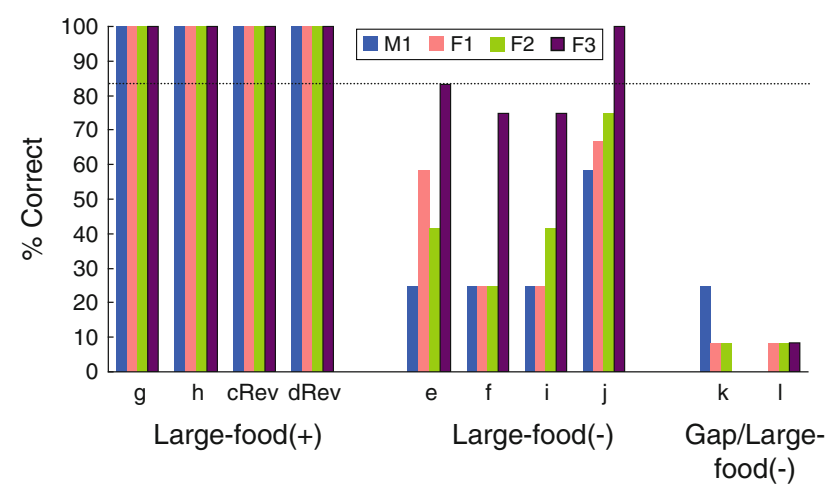

Fig. 6 The percentage of correct choice (i.e., pulling the sheet with a piece of food on its surface) in each problem in Large-food (+), Largefood (-), and Gap/Large-food (-) conditions in Experiment 2, plotted for each subject. The dotted line indicates the line of significance in the binomial test for the 12 trials

problem for the Large-food (+) condition. Except for F3's performance in the Large-food $(-)$ condition (83.33\%), performance for the Large-food (-) condition $(33.33 \%$ (M1), 39.58\% (F1), and $45.83 \%$ (F2)) and the Gap/Largefood (-) condition (12.5\% (M1), 8.33\% (F1), 8.33\% (F2), and $4.17 \%(\mathrm{~F} 3))$ was poor; these success rates were not significant by the binomial test, as shown in Table 2 . To determine whether the distance of the large distracter food affected performance, performance on problems Lf-1 and Lf-2 in Experiment 1 and problems Lf-3 and Lf-4 in Experiment 2 was compared using $t$ tests, but neither of the differences was significant ( $\mathrm{Lf}-1$ vs. Lf-3: $t_{3}=0.27$, $P=0.804 ; \mathrm{Lf}-2$ vs. $\left.\mathrm{Lf}-4: t_{3}=2.35, P=0.134\right)$.

To determine whether there was a learning effect across the sessions (see Fig. 7), a one-way ANOVA was conducted for each type of problem. A significant overall effect was found in the Gap/Large-food $(-)$ condition $\left(F_{5,18}=\right.$ 4.08, $P=0.012$ ), and Tukey's HSD test was significant only for the differences between sessions 3 and 6 $(P=0.014)$ and sessions 5 and $6(P=0.014)$. There was no significant effect in the Large-food $(-)$ condition $\left(F_{5,18}=\right.$ $1.60, P=0.211$ ). An effect of learning was observed in the Gap/Large-food (-) condition. Because large food items

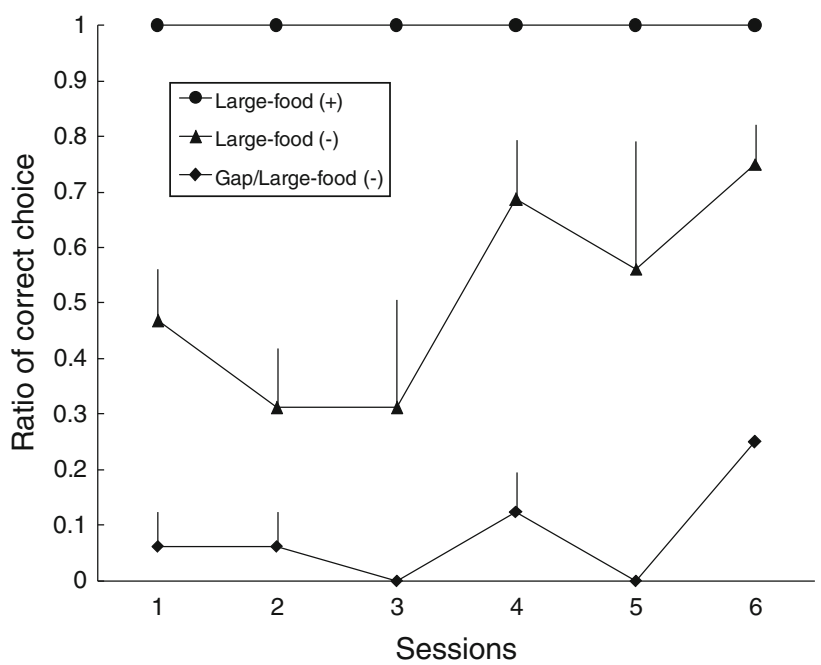

Fig. 7 Averaged percentage of correct choice (i.e., pulling the sheet with a piece of food on its surface) in each test session for each problem in Large-food (+), Large-food (-), and Gap/Large-food (-) conditions in Experiment 2, with the standard error

were both positive and negative in the present experiment, the learning effect observed in the Gap/Large-food (-) condition could not be attributed to non-reinforcement cuing by large food items. However, none of the subjects achieved a significant level of accuracy, even with the learning effects in the Gap/Large-food (-) condition. Thus, the presence of the gap was not a relevant cue within the 24 trials administered. In contrast, no learning effect was found in the Large-food (-) condition in the present experiment, in contrast to the results in Experiment 1. This difference between the experiments is not attributable to the distance from the subject to the large distracter food. Thus, the learning effect across the experiments may have partially affected performance: experience with large but negative food items in Experiment 1 was especially likely to transfer to the early phase of Experiment 2 (Figs. 6, 7).

The proportion of responses in the No-reinforcement condition, in which there was no chance to obtain the food reward (problems NR3/Lf and NR4/Lf), is depicted in Fig. 4. Except for NR3/Lf in F1, binomial tests revealed a

Table 2 Results of the binomial tests for each problem in each subject in Experiment 2

\section{Experiment 2}

\begin{tabular}{|c|c|c|c|c|c|c|c|c|c|c|}
\hline \multicolumn{5}{|c|}{ Large-food (+) } & \multicolumn{4}{|c|}{ Large-food $(-)$} & \multicolumn{2}{|c|}{ Gap + Large-food (-) } \\
\hline Subject & $\mathrm{Lf}+1$ & $\mathrm{Lf}+2$ & $\mathrm{Lf}+3$ & $\mathrm{Lf}+4$ & $\mathrm{Lf}-3$ & $\mathrm{Lf}-4$ & $\mathrm{Lf}-5$ & $\mathrm{Lf}-6$ & $\mathrm{G} 1 / \mathrm{Lf}-$ & $\mathrm{G} 2 / \mathrm{Lf}-$ \\
\hline M1 & $* *$ & $* *$ & $* *$ & $* *$ & & & & & & \\
\hline F1 & $* *$ & $* *$ & $* *$ & $* *$ & & & & & & \\
\hline $\mathrm{F} 2$ & $* *$ & $* *$ & $* *$ & $* *$ & & & & & & \\
\hline F3 & $* *$ & $* *$ & $* *$ & $* *$ & $*$ & & & $* *$ & & $*$ \\
\hline
\end{tabular}

** $P<0.01, * P<0.05$ 
significant difference from the expected performance for all of the problems for each subject, indicating a tendency to direct responses to the sheets with closer, larger food items.

To determine whether the subjects ignored the physical relation of support when the nearby but irretrievable large food item was present in problem $\mathrm{Lf}-5$, we conducted a $t$ test to compare the average numbers of responses to shorter sheets between problems Lf -5 and NR3/Lf in the No-reinforcement condition. The difference was not significant $\left(t_{3}=1.06, P=0.368\right)$. The subjects failed to inhibit their responses when the food items presented were not supported by the sheets (Fig. 4). This tendency was quite similar to the results for problems NR3/f and NR4/f in Experiment 1, although Experiment 2 was conducted after the completion of Experiment 1. Thus, the tendency to reach for the closer food item was not overcome by experience over a relatively short period when the retrievable food item was larger than the irretrievable item.

Thus, when the target food item was much larger than the distracter food item, the marmosets chose the correct sheet without error. In contrast, when the target food item was much smaller than the distracter food item and was located closer to the subjects, their performance was quite poor. In this case, they failed to discriminate based on the perceptual cues of support or contact (as shown by the absence of a significant difference between problems $\mathrm{Lf}-5$ and NR3/Lf) and were affected by a perceptual cue (i.e., size of the food item) that was not relevant to the discrimination between support and contact.

\section{General discussion}

The present experiments show that captive common marmosets are sensitive to differences in the physical relationship of objects in support problems. Their performance was affected by several perceptual aspects of the problems, such as distance between the subject and the food item, saliency of the absence of support, and the size of the distracter food item. The degree to which the subjects were affected by these variables differed greatly. While F2 was the least affected by the distance of the food item, M1 and F1 were affected by distance even after increasing the distance of the irretrievable food item. They made more errors when the distracter food that was not on the sheet was larger than the target food resting on the sheet. This tendency was modified through experience with the same conditions over 12 trials. In contrast, marmosets did not appreciate the presence of a gap between the sheet and the food item even after gaining experience with the problem: they showed a consistent tendency to ignore the separation of the sheet and the food item. Because marmosets develop depth perception early in life, by 4 weeks of age (Whatham and
Judge 2007), and their visual acuity is comparable to that of humans considering the difference in eye size $(30 \mathrm{cpd}$, Ordy and Samorajski 1968; Troilo et al. 1993), the marmosets should have detected the presence of the gap, the difference between the sizes of the food items, the contact and support conditions, and the difference in distance between the food items and the sheets for the two alternatives. Thus, visual capability is not the main explanation for the obtained results.

Performance under the Standard condition in Experiment 1 was comparable to that obtained in a previous study using cotton-top tamarins (Hauser et al. 1999). In the Hauser et al. (1999) study, using 20 trials per session, tamarins were trained to pull correct (reinforced) sheets under similar conditions to problems S1-S8 of the Standard condition in the current study. Although they reached the criterion of 92-100\% accuracy after approximately 20 sessions, accuracy on the first day was around $60 \%$ for subjects starting with the "on problem". In another study (Hauser et al. 2002), tool-trained and tool-untrained tamarins were trained on similar problems, and the numbers of sessions required to meet criterion ( 18 out of 20 or better in two consecutive sessions) were around 5 and 11 , respectively, for "on" and "off" problems, which were similar to problems S1 and S2, respectively, in Experiment 1 of the current study. Thus, it is conceivable that common marmosets and tamarins have a comparable ability to understand the physical causality of contact/support without prior experience with the test problems.

All of the subjects in the current study tended to select the alternative with the closer food item in each trial. Even F3, the only subject that showed significantly better performance than chance in two of the Large-food ( - ) conditions in Experiment 2, performed well only when the correct food item was closer than the incorrect food item (conditions $\mathrm{Lf}-3$ and $\mathrm{Lf}-6$ ). This explains why increasing the distance of the non-reinforced alternative had a positive effect on the marmosets' performance in the Longerdistance condition. Dependency on the perceptual rather than conceptual aspect of support has been observed in other primate species in previous studies (Hauser et al. 1999; Povinelli 2000).

Did the common marmosets in the present study show any indication of conceptual understanding of support? A comparison between problems S7 and NR3/f in Experiment 1 suggested that they likely did not completely ignore the physical relations of support. Additionally, the performance of F2 could be considered as an indication that she might have an elementary concept of support because she showed perfect performance in Experiment 1, including on problems S7 and S7/Ld, in which the irretrievable food item was located closer to her than the retrievable item was. The effects of other variables, such as sizes of the food items, 
may aid in the interpretation of the results. In many of the conditions involving large irretrievable food items, the tamarins could not attend to the relevant aspect of support but were affected by these variables. Given that they may be simultaneously influenced by the knowledge of support and a bias to attempt to obtain rewards that are nearby or large, a failure to solve all configurations of the standard test may not reflect a lack of knowledge. Rather, it may reflect the cumulative effects of different, conflicting strategies and a lack of inhibitory control over the irrelevant strategies, which is assumed to be important in problem-solving tasks (e.g., Diamond 1988; Deacon 1997; Hauser et al. 1999; Evans and Westergaard 2006).

Some of the subjects experienced difficulty in solving the problems in the Large-food (-) conditions and the Gap condition, especially in the early testing sessions (1 and 2). Nonetheless, learning effects were observed in the Large-food (-) condition in Experiment 1, whereas the marmosets consistently showed low accuracy in the Gap conditions. The difference in learning depending on the conditions can be interpreted in terms of the nature of the conditions. In the Large-food (-) condition, there was only one aspect of physical causality that might affect performance; that is, contact or support. In contrast, in the Gap condition, there were two characteristics that might affect marmosets' choices: contact or support and continuity of the supporting sheet. The Gap condition would have been difficult for the subjects because one of the criteria for physical causality was met: namely, the irretrievable food was resting on the discontinuous sheet. Additionally, the problems that did not include a Gap condition required subjects to attend to the edge of the sheet. This might have negatively affected performance in the Gap condition, in which attention should be directed to the middle of the sheets. Such difficulty was also observed in tamarins (Hauser et al. 1999), in which "connected problems" (analogous to the Gap condition in the present study) appeared to be more difficult for the animals than "on problems".

One variable that should be considered is the degree to which the subjects interacted with the external objects during the tests. As shown in the Large-food (-) condition in the present study, which demonstrated that a relatively small amount of experience could change performance, experience outside of the experimental sessions, such as the breeding environment (Furlong et al. 2008) and prior experimental experience (Spaulding and Hauser 2005) affects manipulative knowledge (Hauser et al. 2002) and the degree of neophilia (Day et al. 2003) and, accordingly, test performance. Thus, whether the performance by laboratory-reared common marmosets in the present study represents the general ability of this species remains to be confirmed.
In summary, our common marmosets' understanding of the physical relationship between contact and support is highly susceptible to perceptual variables, proximity of the food to the animal, size of the food, and continuity/discontinuity of the supporting sheet. On the other hand, we observed indications that they did not completely ignore the relevant aspect of support. Thus, although the perceptual model can explain most of the results, marmosets might have knowledge of the difference between support and contact, which would correspond to the intermediate of the perceptual and conceptual understanding models.

Acknowledgments This study complies with the current laws of Japan (Act on Welfare and Management of Animals). All experimental procedures and handling methods were performed in accordance with the "Guidelines of Animal Experimentation" of Keio University and the "Guidelines for Conducting Animal Experiments" of RIKEN. The experiments were approved by the ethics committee of Keio University and the Animal Experiment Committee at RIKEN. The study was supported by the Centre for Advanced Research on Logic and Sensibility, The Global COE Program, at Keio University, Japan, and the Funding Program for World-leading Innovative R\&D on Science and Technology, at RIKEN, Japan. We would like to thank Ms. Chieko Echigo and Mr. Masakado Saeki for running some of the experiments and data collection.

Conflict of interest The authors declare that they have no conflict of interest.

Open Access This article is distributed under the terms of the Creative Commons Attribution Noncommercial License which permits any noncommercial use, distribution, and reproduction in any medium, provided the original author(s) and source are credited.

\section{References}

Abbott DH, Hearn JP (1978) Physical, hormonal and behavioural aspects of sexual development in the marmoset monkey, Callithrix jacchus. J Repro Fert 53:155-166

Araújo A, Arruda MF, Alencar AI, Albuquerque F, Nascimento MC, Yamamoto ME (2000) Body weight of wild and captive common marmosets (Callithrix jacchus). Int J Primatol 21:317-324

Auersperg AM, Gajdon GK, Huber L (2009) Kea (Nestor notabilis) consider spatial relationships between objects in the support problem. Biol Lett 5:455-458

Baillargeon R (1994) How do infants learn about the physical world? Curr Direct Psychol Sci 3:133-140

Burman KJ, Rosa MG (2009) Architectural subdivisions of medial and orbital frontal cortices in the marmoset monkey (Callithrix jacchus). J Comp Neurol 514:11-29

Day RL, Coe RL, Kendal JR, Laland KN (2003) Neophilia, innovation and social learning: a study of intergeneric differences in callitrichid monkeys. Anim Behav 65:559-571

Deacon TW (1997) The symbolic species: the co-evolution of language and the brain. Norton, New York

Diamond A (1988) Differences between adult and infant cognition; is the crucial variable presence or absence of language? In: Weiskrantz L (ed) Thought without language. Clarendon Press, Oxford, UK, pp 337-370

Dias R, Robbins TW, Roberts AC (1996) Dissociation in prefrontal cortex of affective and attentional shifts. Nature 380:69-72 
Elliott R, Deakin B (2005) Role of the orbitofrontal cortex in reinforcement processing and inhibitory control: evidence from functional magnetic resonance imaging studies in healthy human subjects. Int Rev Neurobiol 65:89-116

Evans TA, Westergaard GC (2006) Self-control and tool use in tufted capuchin monkeys (Cebus apella). J Comp Psychol 120:163-166

Ferrari SF (1993) Ecological differentiation in the Callitrichidae. In: Rylands AB (ed) Marmosets and tamarins: systematics, behaviour, and ecology. Oxford University Press, Oxford, UK, pp 314 328

Furlong EE, Boose KJ, Boysen ST (2008) Raking it in: the impact of enculturation on chimpanzee tool use. Anim Cogn 11:83-97

Hauser MD, Kralik J, Botto-Mahan C (1999) Problem solving and functional design features: experiments on cotton-top tamarins, Saguinus oedipus oedipus. Anim Behav 57:565-582

Hauser MD, Santos LR, Spaepen GM, Pearson HE (2002) Problem solving, inhibition and domain-specific experience: experiments on cottontop tamarins, Saguinus oedipus. Anim Behav 64:387396

Hood B, Carey S, Prasada S (2000) Predicting the outcomes of physical events: two-year-olds fail to reveal knowledge of solidity and support. Child Dev 71:1540-1554

Irie-Sugimoto N, Kobayashi T, Sato T, Hasegawa T (2008) Evidence of means-end behavior in Asian elephants (Elephas maximus). Anim Cogn 11:359-365

Mathieu M, Daudelin N, Dagenais Y, Decarie C (1980) Piagetian causality in two house-reared chimpanzees (Pan troglodytes). Can J Psychol 34:179-186

Matsuzawa T (1994) Field experiments on the use of stone tools by chimpanzees in the wild. In: Wrangham RW, McGrew WC, de Waal FBM, Heltne PG (eds) Chimpanzee cultures. Harvard University Press, Cambridge, MA, pp 351-370

Ordy JM, Samorajski T (1968) Visual acuity and ERG-CFF in relation to the morphologic organization of the retina among diurnal and nocturnal primates. Vis Res 8:1205-1225
Piaget J (1952) The origins of intelligence in children (translated by M. Cook.). International Universities Press, New York

Povinelli D (2000) Folk physics for apes: the chimpanzee's theory of how the world works. Oxford University Press, Oxford, UK

Redshaw M (1978) Cognitive development in human and gorilla infants. J Hum Evol 7:133-141

Roberts AC (2006) Primate orbitofrontal cortex and adaptive behaviour. Trends Cogn Sci 10:83-90

Spaulding B, Hauser M (2005) What experience is required for acquiring tool competence? Experiments with two callitrichids. Anim Behav 70:517-526

Spinozzi G, Poti P (1993) Piagetian stage 5 in two infant chimpanzees (Pan troglodytes): The development of permanence of objects and the spatialization of causality. Int J Primatol 14:905-917

Stevens JR, Rosati AG, Ross KR, Hauser MD (2005) Will travel for food: Spatial discounting in two new world monkeys. Curr Biol 15:1855-1860

Troilo D, Howland HC, Judge SJ (1993) Visual optics and retinal cone topography in the common marmoset (Callithrix jacchus). Vis Res 33:1301-1310

Walker SC, Mikheenko YP, Argyle LD, Robbins TW, Roberts AC (2006) Selective prefrontal serotonin depletion impairs acquisition of a detour-reaching task. Eur J Neurosci 23:3119-3123

Walker SC, Robbins TW, Roberts AC (2009) Differential contributions of dopamine and serotonin to orbitofrontal cortex function in the marmoset. Cereb Cortex 19:889-898

Whatham AR, Judge SJ (2007) Rotated prism-wear disrupts emmetropization but does not reliably induce hyperopia in the new world monkey. Vis Res 47:3324-3334

Willatts P (1984) The stage-IV infant's solution of problems requiring the use of supports. Infant Behav Dev 7:125-134

Willatts P (1999) Development of Means-End behavior in young infants: pulling a support to retrieve a distant object. Dev Psychol $35: 651-667$ 